\title{
Iterative Method for Solving a Beam Equation with Nonlinear Boundary Conditions
}

\author{
Quang A. Dang ${ }^{1}$ and Nguyen Thanh Huong ${ }^{2}$ \\ ${ }^{1}$ Institute of Information Technology, VAST, 18 Hoang Quoc Viet, Cau Giay, Hanoi 10000, Vietnam \\ ${ }^{2}$ College of Science, Thai Nguyen University, Thai Nguyen City 23000, Vietnam
}

Correspondence should be addressed to Quang A. Dang; dangqa@ioit.ac.vn

Received 3 June 2013; Accepted 16 July 2013

Academic Editor: Michele Benzi

Copyright (c) 2013 Q. A. Dang and N. T. Huong. This is an open access article distributed under the Creative Commons Attribution License, which permits unrestricted use, distribution, and reproduction in any medium, provided the original work is properly cited.

In this paper, we propose an iterative method for solving a beam problem which is described by a nonlinear fourth-order equation with nonlinear boundary conditions. The method reduces this nonlinear fourth-order problem to a sequence of linear second-order problems with linear boundary conditions. The convergence of the method is proved, and some numerical examples demonstrate the efficiency of the method.

\section{Introduction}

In the paper, we consider the following boundary value problem (BVP):

$$
\begin{gathered}
u^{(4)}(x)=f\left(x, u, u^{\prime}\right), \quad 0<x<L, \\
u(0)=0, \quad u(L)=0, \\
u^{\prime \prime}(0)=g\left(u^{\prime}(0)\right), \quad u^{\prime \prime}(L)=h\left(u^{\prime}(L)\right),
\end{gathered}
$$

which models bending equilibrium of elastic beams on nonlinear supports [1]. Here, $u$ represents the deflection of an elastic beam of length $L$, subjected to a force $f$ exerted by a foundation, and $g$ and $h$ express the influence of fixed torsional springs at the ends of the beam.

In [2], the problem (1) by means of a Green function was reduced to the problem of finding fixed point of a nonlinear integral equation by successive approximations. Therefore, at each iteration, it is needed to compute a single and a double integrals and a derivative. Differently from this approach in this paper following the methodology of $[3,4]$, we lead the solution of problem (1) to a sequence of simple linear boundary value problems (BVPs) for secondorder equation, which are easily solved numerically. The convergence of the iterative method is established by the contraction principle. Some performed numerical examples demonstrate the efficiency of the method.

It should be noticed that the idea of reduction of linear BVPs for fourth partial differential equations, namely, for the biharmonic and biharmonic-type equations to operator equations for investigating iterative methods for their solution was successfully used by ourselves in many works, for example, in $[5,6]$.

\section{Iterative Method}

First, we reduce problem (1) to an operator equation, and then apply the successive approximation method to the latter one. For this purpose, we set

$$
u^{\prime}=v, \quad u^{\prime \prime}=w .
$$

Then, due to the boundary conditions for the function $u$ we have

$$
u(x)=\int_{0}^{x} v(t) d t,
$$

and the function $v$ has the property

$$
\int_{0}^{L} v(t) d t=0 .
$$


By the setting (2), problem (1) is decomposed to the problems

$$
\begin{gathered}
w^{\prime \prime}(x)=f\left(x, \int_{0}^{x} v(t) d t, v(x)\right), \quad 0<x<L, \\
w(0)=g(v(0)), \quad w(L)=h(v(L)), \\
u^{\prime \prime}(x)=w(x), \quad 0<x<L, \\
u(0)=0, \quad u(L)=0 .
\end{gathered}
$$

Obviously, the solution $u(x)$ from these problems depends on the function $v$. Consequently, its derivative $u^{\prime}$ also depends on $v$. Therefore, we can represent this dependence by an operator

$$
u^{\prime}=B v .
$$

Combining with the first relation in (2), we get the operator equation for $v$ :

$$
v=B v .
$$

That is, $v$ is a fixed point of $B$.

Now, we consider properties of the operator $B$. For this purpose, we introduce the space

$$
S=\left\{v \in C([0, L]), \int_{0}^{L} v(t) d t=0\right\} .
$$

Next, we make the following assumptions on the given functions in Problem (1): there exist constants $\lambda_{f}, \lambda_{g}$, and $\lambda_{h}>0$ such that

$$
\begin{gathered}
|f(t, u, v)-f(t, \bar{u}, \bar{v})| \leq \lambda_{f} \max \{|u-\bar{u}|,|v-\bar{v}|\}, \\
|g(u)-g(\bar{u})| \leq \lambda_{g}|u-\bar{u}|, \\
|h(u)-h(\bar{u})| \leq \lambda_{h}|u-\bar{u}|
\end{gathered}
$$

for any $u, \bar{u}, v$, and $\bar{v}$.

Proposition 1. The operator B maps the space $S$ to $S$ and is a contraction operator in $S$ if the number

$$
q=\frac{L^{3}}{16} \lambda_{f} \max \left(\frac{L}{2}, 1\right)+\frac{L}{2}\left(\lambda_{g}+\lambda_{h}\right)<1 .
$$

Proof. Since by definition $B v=u^{\prime}$, then $\int_{0}^{L} B v d t=u(L)-$ $u(0)$. Due to the boundary conditions for $u(x)$, we have $\int_{0}^{L} B v d t=0$; that is, $B v \in S$.

Now, we prove the contraction property of $B$. Let $v, \bar{v}$ be two functions from $S, w, \bar{w}$ and $u$, and let $\bar{u}$ be the corresponding solutions of the problems (5), (6). Then, using Appendix A, it is easy to get

$$
B v-B \bar{v}=u^{\prime}-\bar{u}^{\prime}=\int_{0}^{L}-G_{x}(x, t)[w(t)-\bar{w}(t)] d t .
$$

Consequently, due to the second inequality in (A.4), we have

$$
\|B v-B \bar{v}\|=\left\|u^{\prime}-\bar{u}^{\prime}\right\| \leq \frac{L}{2}\|w-\bar{w}\| .
$$

Here and in sequel, $\|u\|=\max _{0 \leq x \leq L}|u(x)|$. For the solution $w(x)$ of (5) by Appendix A, we have the representation

$$
\begin{aligned}
w(t)= & \int_{0}^{L}-G(t, s) f\left(s, \int_{0}^{s} v(\xi) d \xi, v(s)\right) d s \\
& +\frac{L-t}{L} g(v(0))+\frac{t}{L} h(v(L)) .
\end{aligned}
$$

Therefore,

$$
\begin{aligned}
|w(t)-\bar{w}(t)| \leq & \int_{0}^{L}|G(t, s)| \mid f\left(s, \int_{0}^{s} v(\xi) d \xi, v(s)\right) \\
& \quad-f\left(s, \int_{0}^{s} \bar{v}(\xi) d \xi, \bar{v}(s)\right) \mid d s \\
& +\left|\frac{L-t}{L}(g(v(0))-g(\bar{v}(0)))\right| \\
& +\left|\frac{t}{L}(h(v(L))-h(\bar{v}(L)))\right| .
\end{aligned}
$$

Using the assumptions (10) and Lemma B.1 in Appendix B, we have the estimate

$$
\begin{aligned}
& \|w-\bar{w}\| \\
& \leq \frac{L^{2}}{8} \lambda_{f} \max \left\{\max _{0 \leq s \leq L}\left|\int_{0}^{s}(v(\xi)-\bar{v}(\xi)) d \xi\right|,\right. \\
& \left.\max _{0 \leq s \leq L}|v(s)-\bar{v}(s)|\right\} \\
& \quad+\lambda_{g}|v(0)-\bar{v}(0)|+\lambda_{h}|v(L)-\bar{v}(L)| \\
& \leq\left(\frac{L^{2}}{8} \lambda_{f} \max \left(\frac{L}{2}, 1\right)+\lambda_{g}+\lambda_{h}\right)\|v-\bar{v}\| .
\end{aligned}
$$

Combining this estimate with (13), we obtain

$$
\|B v-B \bar{v}\| \leq\left(\frac{L^{3}}{16} \lambda_{f} \max \left(\frac{L}{2}, 1\right)+\frac{L}{2}\left(\lambda_{g}+\lambda_{h}\right)\right)\|v-\bar{v}\| .
$$

So, under the assumptions (10), the operator $B$ is contractive with the contraction coefficient $q$.

Now, we consider the following iterative method for finding the fixed point of $B$.

(i) Given an initial approximation $v_{0}(x)$, for example, $v_{0}(x)=0$, in $[0, L]$.

(ii) Knowing $v_{k}(x)(k=0,1,2, \ldots)$, solve consecutively two BVPs

$$
\begin{gathered}
w_{k}^{\prime \prime}(x)=f\left(x, \int_{0}^{x} v_{k}(t) d t, v_{k}(x)\right), \quad 0<x<L, \\
w_{k}(0)=g\left(v_{k}(0)\right), \quad w_{k}(L)=h\left(v_{k}(L)\right), \\
u_{k}^{\prime \prime}(x)=w_{k}(x), \quad 0<x<L, \\
u_{k}(0)=0, \quad u_{k}(L)=0 .
\end{gathered}
$$


(iii) Update the new approximation

$$
v_{k+1}(x)=u_{k}^{\prime}(x) .
$$

From the fixed point theorem and (B.2), we have the following.

Theorem 2. Under the assumptions (10) and (11) the iterative method (5), (6) converges with rate of geometric progression with the quotient $q$, and there hold the estimates

$$
\begin{gathered}
\left\|u_{k}^{\prime}-u^{\prime}\right\| \leq \frac{q^{k}}{1-q}\left\|v_{1}-v_{0}\right\|, \\
\left\|u_{k}-u\right\| \leq \frac{L}{2}\left\|u_{k}^{\prime}-u^{\prime}\right\|,
\end{gathered}
$$

where $u$ is the exact solution of the original problem (1).

\section{Numerical Examples}

In order to realize the iterative process, we use the finite difference method [7] for solving BVPs (5) and (6), where we use formulas of second-order approximation for the second derivatives and the trapezium formula for computing definite integral on a uniform grid $\bar{\omega}_{h}=\left\{x_{i}=i h, i=0, \ldots, N\right\}$ with the stepsize $h=1 / N$, where $N+1$ is the number of grid points. For computing the first derivative in (19), we also use formulas of second-order approximation.

For testing the convergence of the method, we perform some experiments for the case of the known exact solutions and also for the case of the unknown exact solutions.

Example 1. We take as in [2]

$$
\begin{gathered}
f(x, u, v)=x^{5}-x^{4}-x^{3}+121 * x-24-u, \\
g(v)=0, \quad h(v)=-2 v .
\end{gathered}
$$

The exact solution in $[0,1]$ is

$$
u(x)=x^{5}-x^{4}-x^{3}+x .
$$

The convergence of the method is presented in Table 1 , where $\|y\|_{\bar{\omega}_{h}}=\max _{0 \leq i \leq N}\left|y_{i}\right|$.

Example 2. We take as in [2]

$$
\begin{array}{r}
f(x, u, v)=4 \pi^{4} \sin (\pi x) \cos (\pi x) \\
-\frac{\sin ^{2}(\pi x) \cos ^{2}(\pi x)}{16}+u^{2}, \\
g(v)=\frac{v}{2}-\frac{\pi}{8}, \quad h(v)=\frac{v}{2}-\frac{\pi}{8} .
\end{array}
$$

The exact solution in $[0,1]$ is

$$
u(x)=\frac{\sin (\pi x) \cos (\pi x)}{4} .
$$

The convergence of the method is presented in Table 2.
TABLE 1: The convergence in Example 1 for the starting approximation $v_{0}=0$.

\begin{tabular}{lccc}
\hline$N$ & $\begin{array}{c}\text { Number of } \\
\text { iterations }\end{array}$ & $\left\|u_{k}-u\right\|_{\bar{\omega}_{h}}$ & $h^{2}$ \\
\hline 80 & 16 & $8.4293 e-5$ & $1.5625 e-4$ \\
100 & 16 & $6.8623 e-5$ & $1.0 e-4$ \\
150 & 18 & $3.2021 e-5$ & $4.4444 e-5$ \\
200 & 20 & $3.586 e-6$ & $2.5 e-5$ \\
250 & 22 & $1.3269 e-5$ & $1.6 e-5$ \\
\hline
\end{tabular}

TABLE 2: The convergence in Example 2 for the starting approximation $v_{0}=0$.

\begin{tabular}{lccc}
\hline$N$ & $\begin{array}{c}\text { Number } \\
\text { of iterations }\end{array}$ & $\left\|u_{k}-u\right\|_{\bar{\omega}_{h}}$ & $2 h^{2}$ \\
\hline 80 & 4 & 0.00026809 & 0.0003125 \\
100 & 4 & 0.000012153 & 0.0002 \\
140 & 4 & $1.0204 e-4$ & $3.5739 e-5$ \\
160 & 4 & $7.8125 e-5$ & $3.0464 e-5$ \\
200 & 4 & $5 e-5$ & $2.7057 e-5$ \\
250 & 4 & $3.2 e-5$ & $2.6849 e-5$ \\
\hline
\end{tabular}

TABLE 3: The convergence in Example 3 for the starting approximation $v_{0}=0$.

\begin{tabular}{lcc}
\hline$N$ & $K_{1}$ & $K_{2}$ \\
\hline 80 & 6 & 6 \\
100 & 6 & 6 \\
150 & 6 & 6 \\
200 & 7 & 7 \\
250 & 7 & 7 \\
\hline
\end{tabular}

In the next examples, the exact solution of Problem (1) is unknown, and we carry out iterations until $\left\|v_{k+1}-v_{k}\right\| \leq h^{2}$.

Example 3. We consider the influence of the right-hand side $f(x, u, v)$ on the solution. For this purpose, we take two functions

$$
\begin{gathered}
f_{1}(x, u, v)=4 \pi^{4} \sin (\pi x) \cos (\pi x)+u^{2}+2 v, \\
f_{2}(x, u, v)=4 \pi^{4} \sin (\pi x) \cos (\pi x)+\frac{u^{2}}{2}+v^{2}+u v,
\end{gathered}
$$

with the same

$$
g(v)=\frac{v^{2}}{2}, \quad h(v)=\frac{v^{3}}{5} .
$$

The convergence of the iterative method in these cases is given in Table 3, where $K_{1}$ and $K_{2}$ are the number of iterations for the cases of $f_{1}$ and $f_{2}$, respectively. The graph of the approximate solutions for these cases is depicted in Figure 1. 


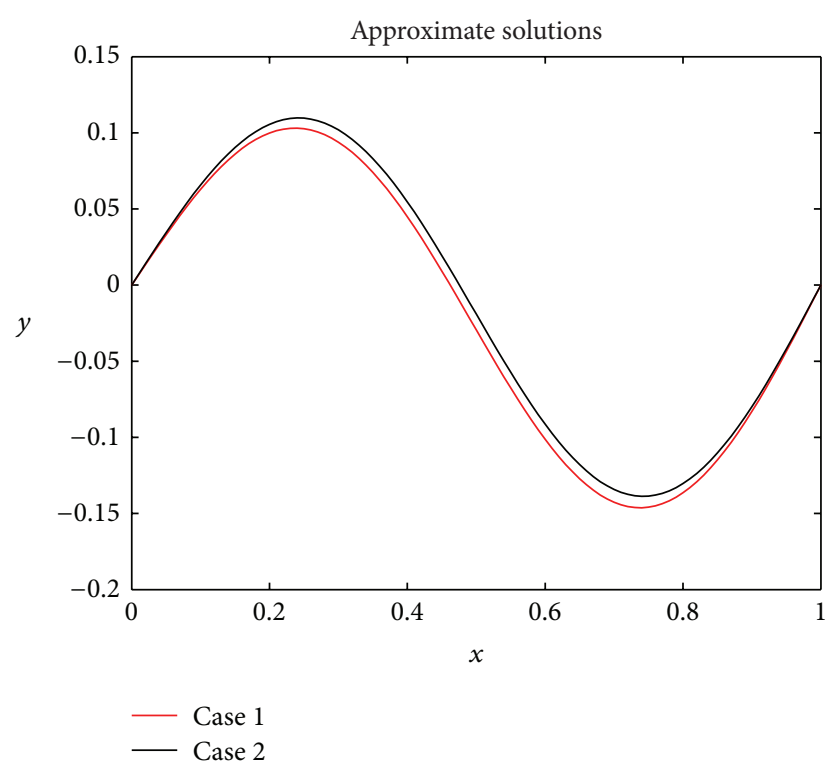

FIGURE 1: Graph of approximate solutions in Example 3 for the cases $f_{1}$ and $f_{2}$.

TABLE 4: The convergence in Example 4 for the starting approximation $v_{0}=0$.

\begin{tabular}{lcc}
\hline$N$ & $K_{1}$ & $K_{2}$ \\
\hline 80 & 6 & 6 \\
100 & 6 & 6 \\
150 & 7 & 6 \\
200 & 7 & 7 \\
250 & 7 & 7 \\
\hline
\end{tabular}

Example 4. We consider the influence of the functions $g$ and $h$ in boundary conditions on the solution. For this purpose we take

$$
f(x, u, v)=4 \pi^{4} \sin (\pi x) \cos (\pi x)+u^{2}+2 v,
$$

and two combinations of $g(v)$ and $h(v)$

$$
\begin{array}{cc}
g_{1}(v)=v^{2}+1, & h_{1}(v)=\frac{v}{2}-\frac{\pi}{8}, \\
g_{2}(v)=\frac{v^{2}}{2}, & h_{2}(v)=\frac{v^{3}}{5} .
\end{array}
$$

The convergence of the iterative method in these cases is given in Table 4, where $K_{1}$ and $K_{2}$ are the number of iterations for the cases of $g_{1}, h_{1}$ and $g_{2}, h_{2}$, respectively. The graph of the approximate solutions for these cases is depicted in Figure 2.

\section{Concluding Remarks}

In this paper, we propose an iterative method for solving a nonlinear beam equation with nonlinear boundary conditions. The idea of the method is the reduction of the problem for the fourth-order equation to a sequence of linear secondorder problems, which are easily solved numerically. The

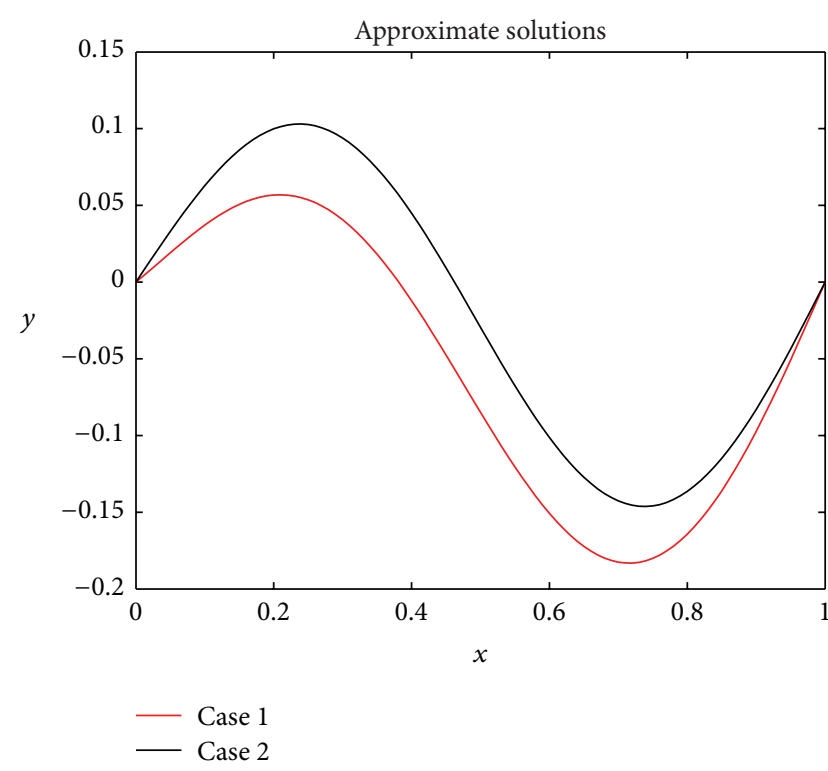

FIGURE 2: Graph of approximate solutions in Example 4 for different pairs of $g, h$.

method can be successfully applied to other nonlinear beam problems, for example, the problems considered in [8-10]. For the problems in $[8,9]$, the nonlinear BVPs are reduced to sequences of Cauchy problems for linear second-order ODE. The application of the proposed method to these problems will be investigated in the future.

\section{Appendices}

\section{A. Green Function and Its Estimates (See [2])}

The Green function associated to the second-order problem

$$
\begin{array}{cc}
u^{\prime \prime}(x)=v(x), & 0<x<L, \\
u(0)=\alpha, & u(L)=\beta,
\end{array}
$$

is

$$
G(x, t) \begin{cases}\frac{x(L-t)}{L}, & x \leq t \leq L, \\ \frac{t(L-x)}{L}, & t \leq x \leq L .\end{cases}
$$

So, the solution of (A.1) is represented in the form

$$
u(x)=\int_{0}^{L}-G(x, t) v(t) d t+\alpha \frac{L-x}{L}+\beta \frac{x}{L} .
$$

For the function Green, there hold the estimates

$$
\int_{0}^{L}|G(x, t)| d t \leq \frac{L^{2}}{8}, \quad \int_{0}^{L}\left|G_{x}(x, t)\right| d t \leq \frac{L}{2} .
$$




\section{B. Norm in $C^{1}([0, L])$}

In the space

$$
E=\left\{u \in C^{1}([0, L]), \quad u(0)=u(L)=0\right\},
$$

there holds the estimate

$$
\|u\| \leq \frac{L}{2}\left\|u^{\prime}\right\|
$$

where $\|u\|=\max _{0 \leq x \leq L}|u(x)|$ (see [2]).

Lemma B.1. For any function $v(x)$ from the space $S$ defined by (9), there holds the estimate

$$
\max _{0 \leq s \leq L}\left|\int_{0}^{s} v(x) d x\right| \leq \frac{L}{2}\|v\|_{\infty} .
$$

Indeed, it is valid because for this function $v$ if setting

$$
\varphi(s)=\int_{0}^{s} v(t) d t
$$

then we have

$$
\varphi(0)=\varphi(L)=0, \quad \varphi^{\prime}(s)=v(s),
$$

and (B.3) follows immediately from (B.2).

\section{References}

[1] J. H. Ginsberg, Mechanical and Structural Vibrations, John Wiley and Sons, New York, NY, USA, 2001.

[2] E. Alves, E. Toledo, L. Pereira Gomes, and M. Souza Cortes, "A note on iterative solutions for a nonlinear fourth order ode," Boletim da Sociedade Paranaense de Matemática, vol. 27, no. 1, p. 1520, 2009.

[3] Q. A. Dang and V. T. Luan, "Iterative method for solving a nonlinear fourth order boundary value problem," Computers and Mathematics with Applications, vol. 60, no. 1, pp. 112-121, 2010.

[4] Q. A. Dang, V. T. Luan, and D. Q. Long, "Iterative method for solving a fourth order differential equation with nonlinear boundary condition," Applied Mathematical Sciences, vol. 4, no. 69-72, pp. 3467-3481, 2010.

[5] Q. A. Dang, "Iterative method for solving the Neumann boundary value problem for biharmonic type equation," Journal of Computational and Applied Mathematics, vol. 196, no. 2, pp. 634-643, 2006.

[6] Q. A. Dang and T. S. Le, "Iterative method for solving a problem with mixed boundary conditions for biharmonic equation," The Advances in Applied Mathematics and Mechanics, vol. 1, no. 5, pp. 683-698, 2009.

[7] A. A. Samarskii, The Theory of Difference Schemes, Marcel Dekker, New York, NY, USA, 2001.

[8] T. F. Ma and J. Da Silva, "Iterative solutions for a beam equation with nonlinear boundary conditions of third order," Applied Mathematics and Computation, vol. 159, no. 1, pp. 11-18, 2004.

[9] T. F. Ma, "Positive solutions for a nonlocal fourth order equation of Kirchhoff type," Discrete and Continuous Dynamical Systems, pp. 694-703, 2007.

[10] T. F. Ma and A. L. M. Martinez, "Positive solutions for a fourth order equation with nonlinear boundary conditions," Mathematics and Computers in Simulation, vol. 80, no. 11, pp. 2177-2184, 2010. 


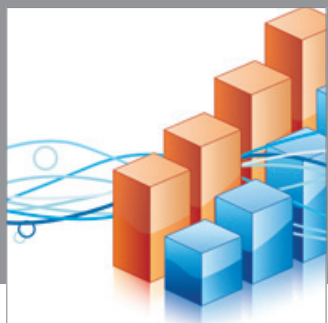

Advances in

Operations Research

mansans

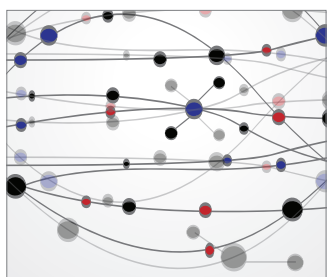

The Scientific World Journal
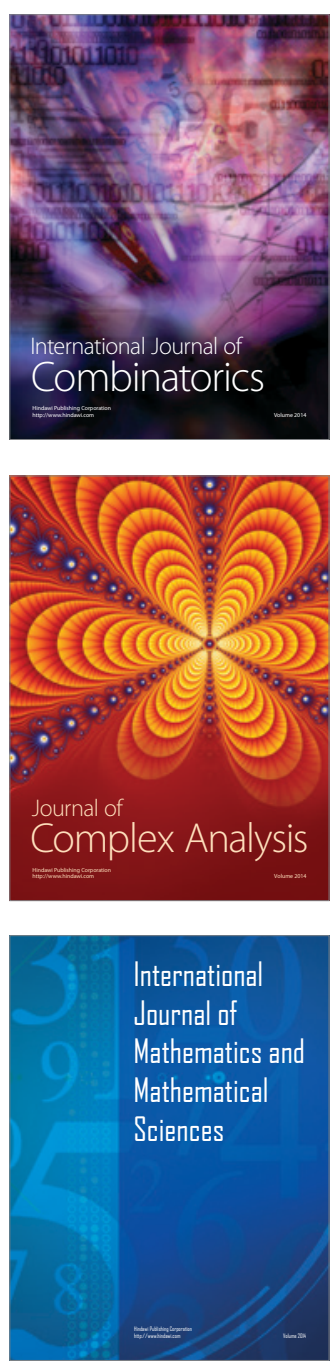
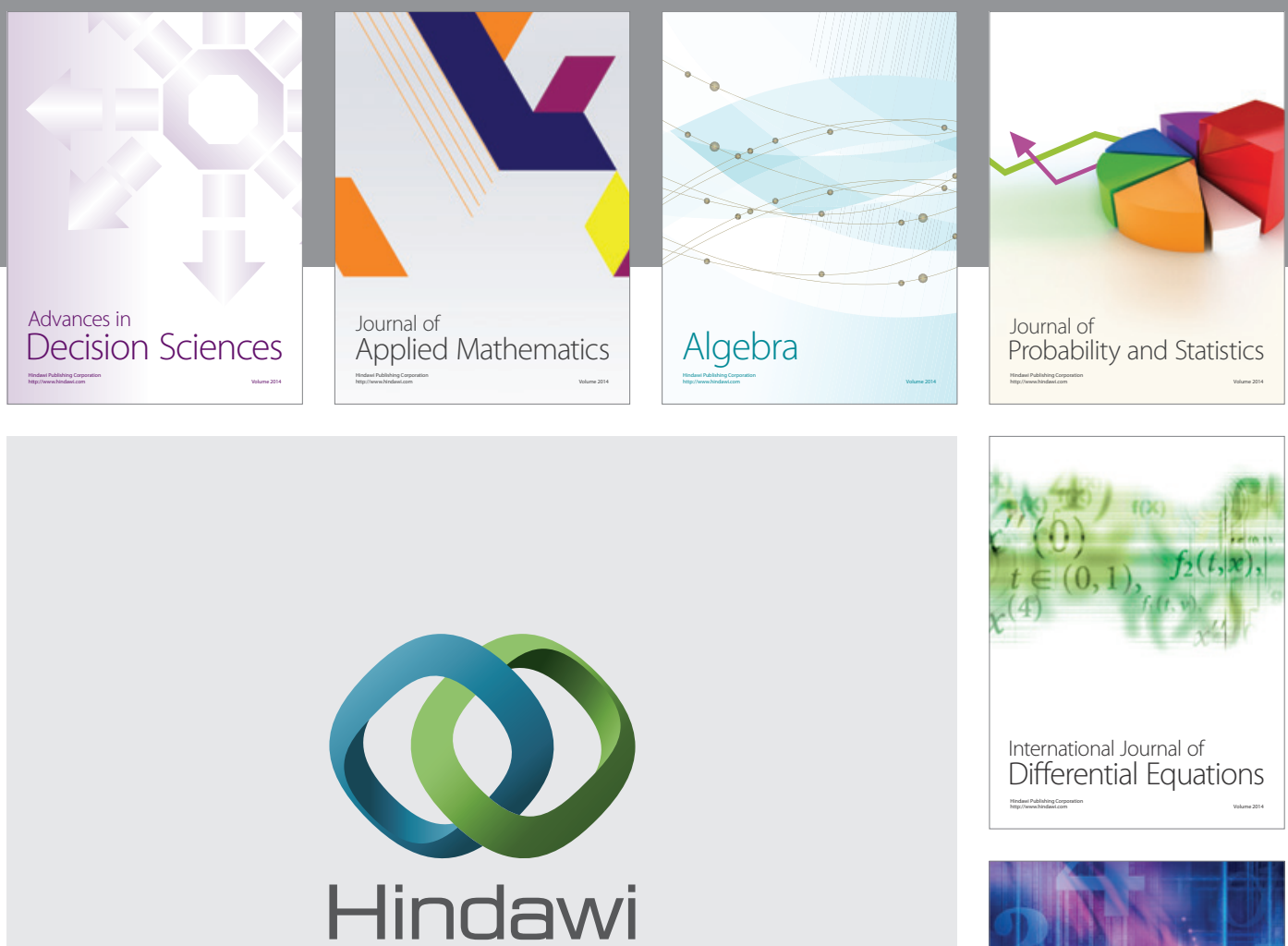

Submit your manuscripts at http://www.hindawi.com
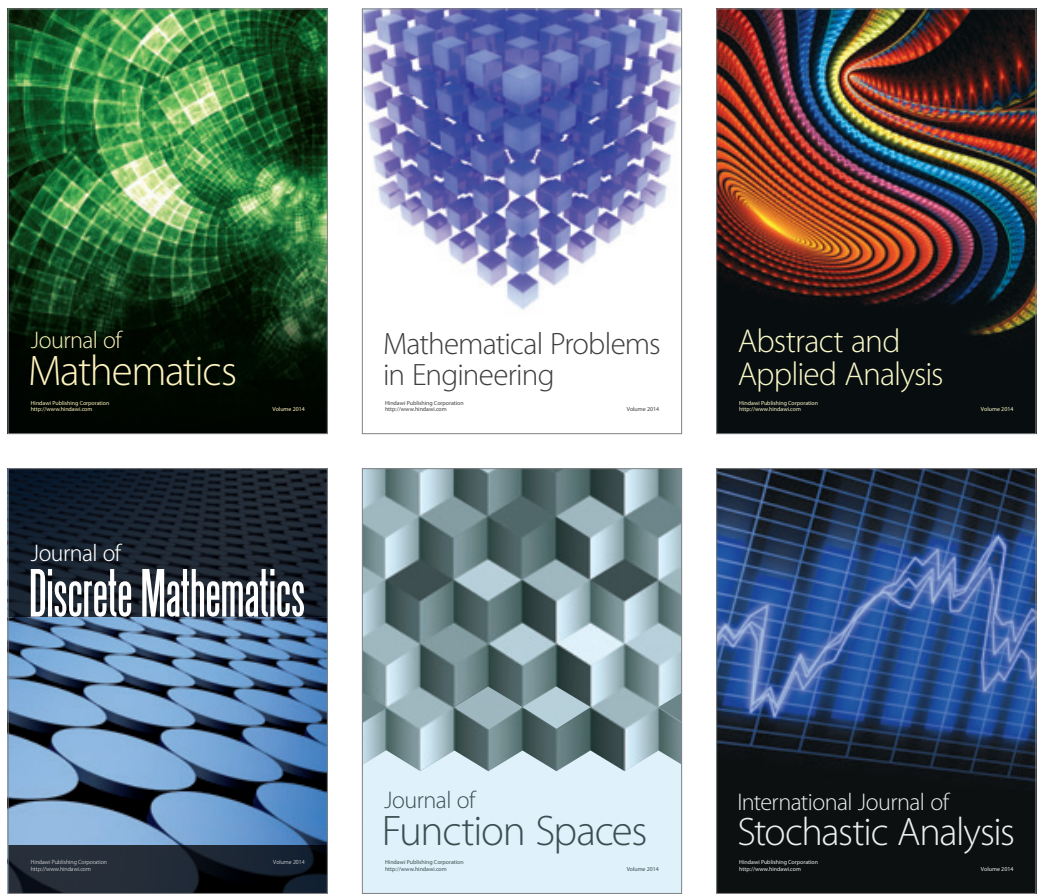

Journal of

Function Spaces

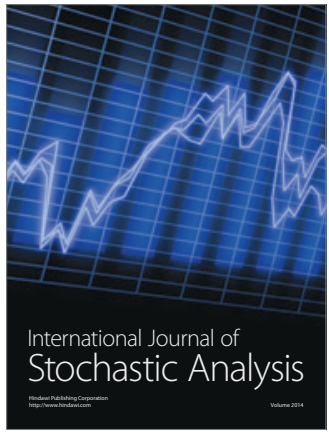

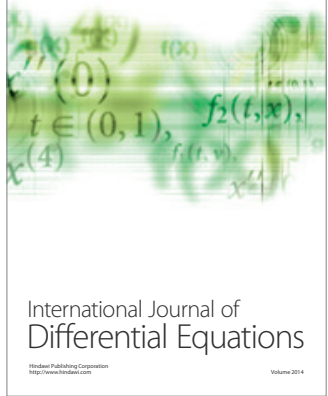
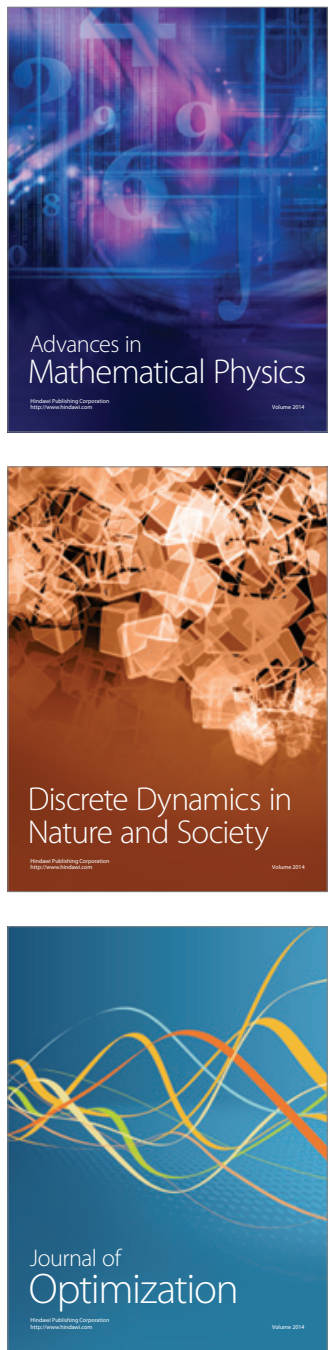